\title{
The Effect of DOTS Strategy on the Transmission of Tuberculosis with Endogenous Reactivation and Exogenous Reinfection Factors
}

\author{
Nursanti Anggriani $^{* 1}$, Islami Nur Fithriati ${ }^{2}$, Dwi Agustian ${ }^{3}$ and Asep K. Supriatna ${ }^{4}$
}

\begin{abstract}
Tuberculosis or TB is one of dangerous infectious diseases in many countries. It is caused by the Mycobacterium tuberculosa bacterium which spreads through the air. One of the methods used to prevent TB is via the introduction DOTS strategy by the WHO. This strategy focuses its concern in the direct observation of the patient in taking their medicine, to make certain that they finished their treatment completely. As time goes by, more complex problems arise related to the treatment of tuberculosis, for example the appearence of endogenous reactivation and exogenous reinfection, and the treatment for the extrapulmonary TB. This study constructs three dynamic models of the TB transmission, for the following situations : i.e. a model without DOTS strategy and the extrapulmonary treatment, a model without DOTS strategy but with treatment, and a model with DOTS strategy which aims to know the effectiveness of DOTS strategy in terms of TB prevention. Based on the analysis resulting from the models, the model with DOTS strategy was proved to be able to reduce the number of TB infection and increase the recovery number from the Tb infection. Therefore, DOTS strategy works very effectively in controlling the transmission of the TB.
\end{abstract}

Keywords - Tuberculosis (TB), DOTS strategy, dynamic model.

\section{INTRODUCTION}

$\mathrm{S}$ INCE 1993, WHO (World Health Organization) states that tuberculosis is a global emergency for humans. With the progress that has been achieved until 2012, it is estimated there are still 9 million new tuberculosis cases and 1.4 million people died from tuberculosis [3]. Tuberculosis or TB is a type of infectious disease caused by the bacterium Mycobacterium tuberculosa that usually attacks the lungs and is spread through air body. In 19921993, the WHO Global Tuberculosis Programme introduces a $\mathrm{Tb}$ control strategy that became known as the DOTS strategy (Directly Observed Treatment Shortcourse). This strategy focuses attention on monitoring patients in taking medicine and ensure that patients carry out the treatment to completion. The aim of this strategy is to prevent the occurrence of bacterial resistance to anti-

Nursanti Anggriani ${ }^{1}$ is with Department of Mathematics Universitas Padjadjaran

Islami Nur Fithiati ${ }^{2}$, was with Department of Mathematics Universitas Padjadjaran

Dwi Agustian ${ }^{3}$, is with Department of Medicine Universitas Padjadjaran Asep K. Supriatna, is with Department of Mathematics Universitas Padjadjaran tuberculosis drugs (OAT) so as to reduce the number of $\mathrm{Tb}$ infection.

Models of treatment of tuberculosis using DOTS strategy derived from [2] with the development in their comparison of the three conditions of the model, ie without the DOTS strategy without treatment, without the DOTS treatment strategy, and with the DOTS strategy. While research by exogenous reinfection and endogenous reactivation obtained from research SO Adewale, C.N. Podder, and A.B. Gumel [1] and then developed into a dynamic model implementation of the DOTS strategy by endogenous and exogenous reinfection on the reactivation of pulmonary TB and extra-pulmonary tuberculosis.

In this paper, we make the model of $\mathrm{TB}$ with endogenous and exogenous reinfection factor, we construct three model, there are the model without DOTS and treatment, the model without DOTS with treatment and the last with DOTS and treatment. We will show the effect of DOTS strategy by numerical analysis.

\section{II.RESULT AND DISCUSSION}

In this paper, we have three model, there are TB model Without DOTS and Treatment, without DOTS and with treatment and with DOTS and treatment. In this model, the population of individuals is devided into six compartment, there are susceptible population (S), latently infected population (L), the population of individuals that infected with pulmonary $\mathrm{Tb}\left(\mathrm{I}_{\mathrm{i}}\right)$, the population of individuals that infected with extrapulmonary $\mathrm{Tb}\left(\mathrm{I}_{\mathrm{n}}\right)$, the populations that have recovered from pulmonary $\mathrm{Tb}$ infection $\left(R_{i}\right)$, and the last is the populations that have recovered from extrapulmonary $\mathrm{Tb}$ infection $\left(R_{n}\right)$. We also assume that the population is constant, birth rate and mortality rate is the same. The following numerical examples discuss the comparison of three model and dynamic for each compartment. Figure 1-3 shows the dynamic of compartment Susceptible, Laten, Infectious Tb, Non Infectious Tb, Recovered (Infectious) and Recovered (non infectious). 


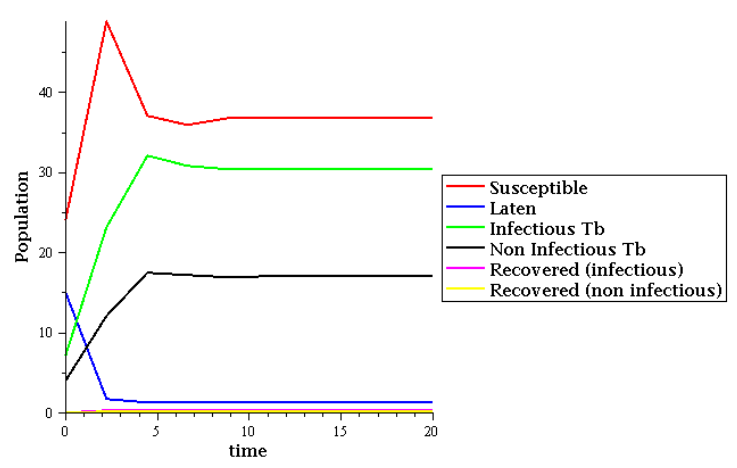

Fig. 1. Graph for the model without DOTS Strategy and without treatment. The value of parameters that used in this model are $\pi=50 ; \mu=0,2509710694 ; \kappa=0,2522 ; \gamma_{\mathrm{i}}=0,12 ; \gamma_{\mathrm{n}}=$ 0,$11 ; \psi_{\mathrm{L}}=\psi_{\mathrm{i}}=\Psi_{\mathrm{n}}=0,85 ; \omega_{1}=0,16 ; \omega_{2}=0,78 ; \delta=$ 0,$02883022718 ; \mu_{n}=0,5 ; \mu_{i}=0,6587000003 ; \lambda_{n}=$ 0,$5 ; \lambda_{\mathrm{i}}=0,7100995912 ; \beta_{\mathrm{i}}=0,0027308696 ; \beta_{\mathrm{n}}=$ 0,005

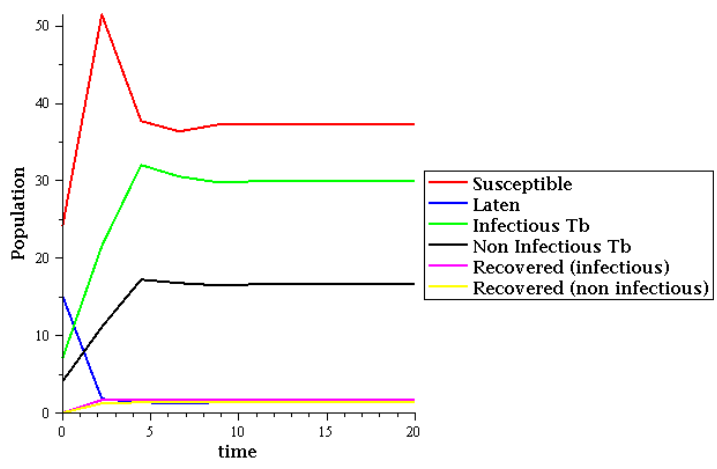

Fig. 2. Graph for the model without DOTS Strategy with treatment. The value of parameters that used in this model are $\gamma_{\mathrm{i}}=0,22 ; \gamma_{\mathrm{n}}=0,21 ; \mathrm{c}=0,7883184433$ and the value for the other parameters are equal with the value of parameters in Figure 1.

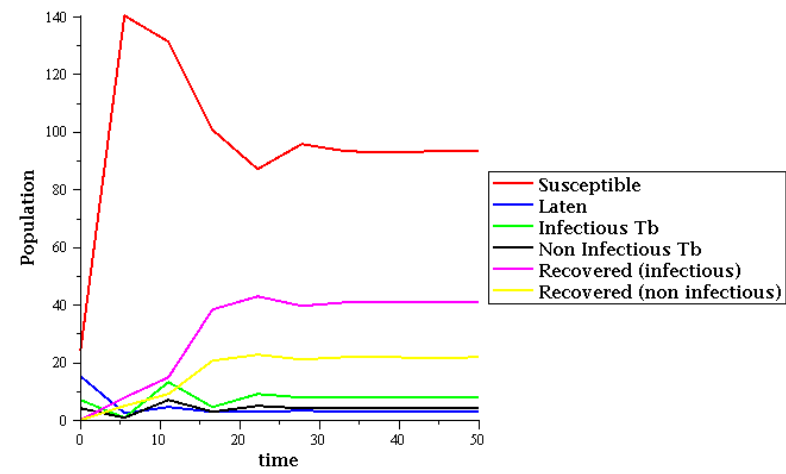

Fig. 3. Graph for the model with DOTS Strategy. The value of parameters that used in this model are

$\gamma_{\mathrm{i}}=0,32 ; \gamma_{\mathrm{n}}=0,31 ; \mathrm{c}=0,9883184433$ and the value for the other parameters are equal with the value of parameters in Figure 1.

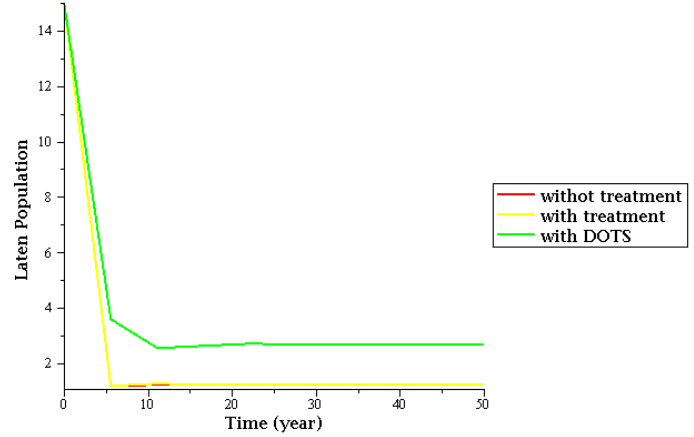

Fig 4. Comparison Chart of Three Models for Latent Compartment

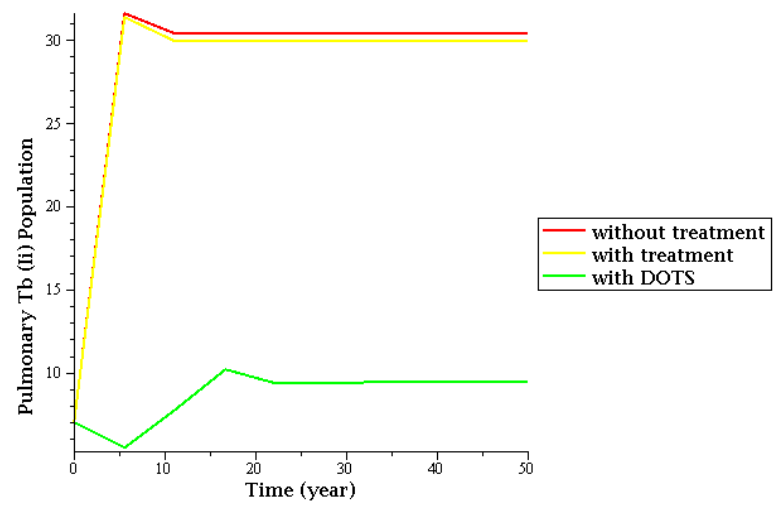

Fig. 5. Comparison Chart of Three Models for Pulmonary Tb Compartment

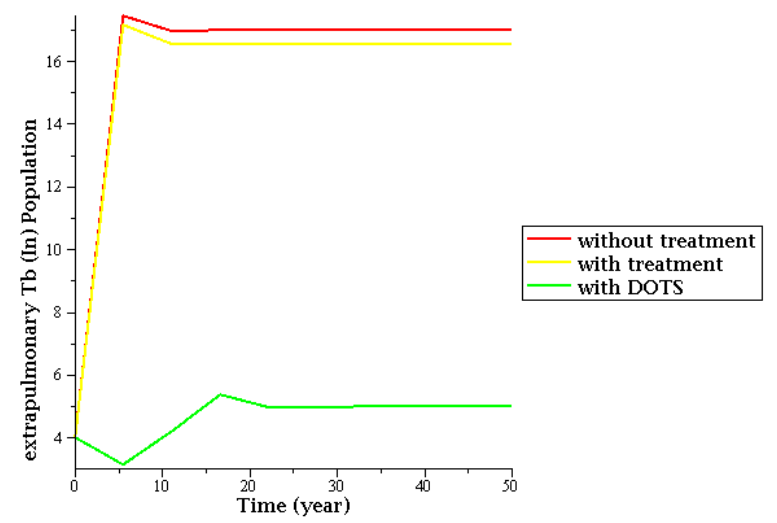

Fig. 6. Comparison Chart of Three Models for extrapulmonary Tb Compartment

From the graph on Figure 5 and 6, it can be seen that for both infectious and non infectious compartment, the number of individuals on the model with the implementation of the DOTS strategy has a smaller value than the other two models, which means the DOTS strategy is effective to reduce the number of pulmonary $\mathrm{Tb}$ infection.

Based on the analysis and graphs obtained from the model of disease control tuberculosis with three conditions, it can be concluded that the DOTS strategy is effectively used to control TB disease compared to no application of the DOTS strategy (either with or without treatment), because of the DOTS strategy is able to reduce the number $\mathrm{Tb}$ infection and increase the cure rate of TB infection. In addition, the DOTS strategy is also effectively used for 
controlling tuberculosis, both $\mathrm{Tb}$ and $\mathrm{Tb}$ extrapulmonary lung, and by including reactivation of endogenous factors and exogenous reinfection. Lastly, a small change of some parameter value proved to be very influential on the graph model of tuberculosis disease control with three conditions, so it can be said that the model is quite sensitive to changes of some relevant parameter values.

\section{ACKNOWLEDGMENT}

This work is funded by the ALG (Academic Leadership Grant) of Universitas Padjadjaran.

\section{REFERENCES}

[1] Adewale Adewale, S.O., Podder, C.N., dan Gumel, A.B. 2009. Mathematical Analysis of a TB Transmission Model with DOTS. Canadian Applied Mathematics Quarterly, Vol. 17, No. 1

[2] Laxminarayan, R., Klein, E., Dye, C., Floyd, K., Darley, S., dan Adeyi, O. 2007. Economic Beneft of Tuberculosis Control. The World Bank.

[3] World Health Organization. 2013. Global Tuberculosis Report 2013. 